\title{
1. Complex Science for a Complex World: An Introduction
}

\author{
Pascal Perez and David Batten
}

\section{Introduction}

It is well known that human activities are endangering the stability and sustainability of many fragile ecosystems to such an extent that their future is in doubt. At the same time, these ecosystems are inherently challenging to manage successfully because of the complexity and uncertainty associated with their ongoing evolution. Much of this complexity and uncertainty may be attributed to the human dimension. Thus it is imperative that we deepen our understanding of how and why people choose to interact with one another and how this interactive behaviour affects these ecosystems as time passes. This book is a small contribution in this direction. It examines ways in which the collective behaviour of human beings can vary, and how this behaviour may affect the natural ecosystems which humans enjoy.

Fortunately, a new kind of science is helping us deepen our understanding of how human ecosystems might grow and change over time. Beyond a mere collation of various reflections and applications, the chapters in this book aim to convince the reader that this new kind of science is worthy of our attention. It is a science that fully embraces the complexity of our surrounding world. It is also a science that addresses the frontiers of interactions between human behaviour and environmental responses. Furthermore, it is a science that challenges our limited understanding and treatment of uncertainty. And, finally, because it is socially embedded, it is a science that can generate partnerships with local communities in a constructive manner.

The computational science we describe and discuss in this book is sufficiently different to normative science that we may call it a new kind of science. Observation and experimentation are still alive and well, but they have been joined by an entirely new breed of computational science: simulation. However, it should be recognised that science - old or new - is not a process for finding absolute truths or universal laws. As Roger Bradbury argues persuasively in the next chapter, science is not just a process, but is really a system-a complex adaptive system.

The complex adaptive system known as science consists of the scientists or agents and the knowledge system that they accumulate by way of an interactive and ongoing exchange of ideas. As Bradbury notes, the former is essentially a 
social system, whereas the latter is a playground of memes - with the interaction strongly mediated by the recipe. Science is a dialogue between humankind and nature, the results of which have been - and will continue to be - unpredictable (Prigogine 1996: 153). Like science, a human ecosystem is a complex adaptive system involving people, other living entities, an environment, information exchange and the co-evolution of all of these things over time. Although a human ecosystem's future state has always been unpredictable, the critical players (agents) on this adaptive stage today are the human participants. Thus it is imperative that we begin to understand human reasoning and its idiosyncrasies.

\section{The complex world of human ecosystems}

\section{Understanding human behaviour}

\section{Deductive versus inductive reasoning}

How do people make decisions in complicated situations? Many psychology texts argue that human reasoning is deductive. Deduction is reasoning from the general to the particular. A perfectly logical deduction yields a conclusion that must be true provided that its premises are true. Thus it involves specifying a set of axioms and proving consequences that can be derived from those premises. In reality, deduction is handy for solving a host of theoretical problems and a handful of simple practical problems, but it is less helpful for tackling complicated practical problems like those associated with the management of human ecosystems. Moreover, it is definitely of no help when the managers involved are likely to behave and react differently over time.

The truth of the matter is that each of us is a unique product of our own brain and our uniquely individual experiences. Our personal knowledge is honed by the concepts, notions and models which we choose to use to represent it. All of this has to be created, put together and revised over time by us as well as by others in society as a whole. Learning has both an individual and a collective dimension, which builds adaptively on the inductive and intuitive skills of a heterogeneous collection of minds. Erwin Schrödinger summed it up well:

The world is a construct of our sensations, perceptions, memories. It is convenient to regard it as existing objectively on its own. But it certainly does not become manifest by its mere existence. Its becoming manifest is conditional on very special goings-on in very special parts of this very world, namely on certain events that happen in a brain.

(Schrödinger 1967: 94)

As a matter of fact, human agents do more than make rational choices by way of simple deduction. In most cases, no one agent knows what all the others are doing, being forced to rely on a limited amount of shared information and unique 
experiences of its own. Hence, they make decisions on the basis of whims, hunches, heuristics or mental models and are willing to modify their preferred mental models (to a greater or lesser extent) and come up with new ones where necessary. In other words, when facing open-ended situations, human agents reason inductively.

In chapter 3, Pascal Perez draws upon philosophy of mind and semiotics to suggest, along with an increasing number of colleagues, that these contextual cognitive processes have deceived attempts to develop a predictive modelling formalism so far. He argues that the Artificial Intelligence paradigm needs to be replaced with more modest, but more robust approaches. Anne Dray and colleagues (chapter 12) provide a meaningful example of such innovative approaches through their description of a companion modelling experience in the South Pacific.

\section{Sheep versus explorers}

Inductive reasoning places different demands on we thinking individuals than the deductive metaphor. It involves pattern formation and pattern recognition, aided by intuition and creativity. Clearly some people are more intuitive or creative than others. They are better at seeking and discovering novel solutions to problems, being willing to experiment, adapt and instigate change. Others merely follow existing patterns, often resisting change under almost any circumstances. Like the spectrum of light, cognitive equipment consists of a mixture of cognitive skills of varying intensities.

We may classify agents in terms of two extreme forms of behaviour: those who actively search for new possibilities are explorers, and those who prefer to remain with the status quo are sheep. The above mentioned spectrum of cognitive skills implies that we all possess sheep and explorer qualities, albeit in different doses. Pure explorers tend to be imaginative, creative, highly-strung individuals who constantly search for better solutions to the problems they face. They are more inclined to reason inductively, to learn quickly and to adapt willingly to changing circumstances. Sheep are more placid, patient and resigned than explorers. Preferring to reason deductively, they are prone to choosing a well established pattern. They mostly cling to particular beliefs because they have worked well in the past. Sheep are slow learners who must accumulate a record of failure before discarding their favourite beliefs.

In chapter 4, David Batten explores sheep and explorer strategies among fishing fleets searching for profitable fishing zones. Non-equilibrium systems scientists such as Peter Allen, having studied the behaviour of fishing fleets, call the sheep Cartesians and the explorers Stochasts. As Allen notes, the first group makes good use of information, but the second generates it. 


\section{Self-referential situations}

Conventional economic wisdom claims that agents have only one reasoning skill: the ability to process the information available to them in a purely logical, deductive manner to arrive at the best decision in a given situation. But this is useless in a self-referential situation like a stock market, where the best thing to do depends on what everyone else is doing. Self-referential situations often arise in human ecosystems, but are rarely recognised as such. A self-referential situation is one in which the forecasts made by the agents involved serve to create the world they are trying to forecast (Batten 2000). In many human decision-making situations, there is no optimal predictor. The best thing each agent can do is to apply the predictor that has worked best so far, then to be willing to re-evaluate the effectiveness of his set of predictors, and to adopt better ones as new information becomes available.

In chapter 4, David Batten develops the mechanisms of such 'perpetual experiments' and their consequences in terms of the collective behaviour of what he calls self-defeating systems. Drawing upon the powerful metaphor proposed by Brian Arthur (1994), the El Farol bar problem, the author suggests that many human ecosystems fall into this category and offer agent-based modelling an unchallenged field of exploration. To a certain extent, Ryan McAllister and colleagues (chapter 14) illustrate the ill-fated consequences of such self-defeating systems in the case of privately-owned pastoral regimes in Australian rangelands.

\section{Complexity of human ecosystems}

\section{Adapting to co-evolution}

Human ecosystems constitute a subset of complex adaptive systems. They correspond to real life systems characterised by very strong and long-term interactions between human communities and their environment. According to John Holland (1995), these systems display the following characteristics:

- Emergence: a system-level phenomena is emergent if it requires new categories to describe it, which are not required to describe the behaviour of the underlying components. In other words, interactive individual components instantiate emerging patterns at the level of the system.

- Path dependency: due to the highly non-linear relationships between individual components or parts of the system, a given system-level phenomena can be achieved, in theory, through an infinite number of combinations at the micro-level.

- Non state equilibrium: the Complex Adaptive Systems display an everchanging dynamic equilibrium, driving back and forth the system between chaotic to ordered states. On the edge of chaos, these systems are very sensitive to any perturbation from the individual components. 
- Adaptation: the evolution of the system is driven by the co-evolution of its individual components. They adapt to their environment and modify it in a recursive way. If the components are cognitive beings, the adaptation relies mainly on the individual and collective learning processes.

According to Stepp et al. (2003), human ecosystems not only process matter and energy flows, but - more specifically-information flows as well. Therefore, they display very specific characteristics. As stated in the previous section, human ecosystems are inherently complex and adaptive, due to the ability of human beings to switch from rational deductive reasoning to inductive pattern recognition. Besides, our ability to communicate and learn from others creates the conditions for co-evolutionary processes in which positive feedback loops follow negative ones, punctuation dispels equilibrium, chaos threatens order, and chance gives a hand to necessity. In chapter 2, Bradbury argues that, until recently, human beings had been able to adapt to changes and to cope with coevolution through rather simple heuristics. But human activities have gradually strengthened the links - let's call it globalisation-between loosely connected environments and societies. The author suggests that:

the balance of [our] effective adaptive strategies has shifted decisively and forever from heuristics to what we might call symbioses - the sorts of strategies that evolution favors in closely connected systems, the ones we see today inside cells and organisms, and between symbiots. (chapter 2, p. 23)

It is indeed remarkable to observe the analogies between what is known as human body functional integrity and what is nowadays called environmental sustainability. More information, more interactions, and shorter communication paths tend to create what David Newth refers to as small worlds in his network-centric analysis of social interactions (chapter 5). The symbiotic dimension of our current understanding of sustainable development is illustrated in two contrasted chapters of this book. In chapter 7, Katherine Daniell and colleagues present a tentative framework to assess sustainable urban development. Taking Christie Walk housing development in Adelaide (Australia) as a case study, the authors insist on the necessity of taking into account water distribution, gas emission, ecosystem health, waste management, economic viability, and social aspiration in order to derive relevant and interrelated sustainability indicators. In chapter 12, Anne Dray and colleagues describe the same necessity of taking into account complex social and spatial interactions in order to help local stakeholders to selfdesign sustainable water management on the atoll of Tarawa (Republic of Kiribati). 


\section{Coping with uncertainty}

By admitting that many human ecosystems are complex and adaptive, we accept their inherent uncertainty. Indeed, if the system is sufficiently complex, it may not be practical or perhaps even possible to know the details of each local interaction. Obviously, the understanding of system-level patterns is often purchased at a cost:

the observer must usually give up the hope of understanding the workings of causation at the level of individual elements.

(Lansing 2003: 185)

As a matter of fact, uncertainty in human ecosystems can result from two different causes: unpredictable non-linear interactions or ill-defined predicates. The latter - more frequently encountered than usually admitted - relies on our limited ability to infer robust causality links among given sets of elementary processes. For example, Durkheim (1979: 58), in his famous study of suicide, concluded that no matter how much a researcher knows about a collection of individuals:

It is impossible to predict which of them are likely to kill themselves. Yet the number of Parisians who commit suicide each year is even more stable than the general mortality rate.

A process that seems to be governed by chance when viewed at the level of the individual turns out to be strikingly predictable at the level of society as a whole. One would argue that statistics prevail in this case, others would invoke Richard Dawkin's memes (Dawkins 1976), but we could also admit that we don't know enough yet about the intimate social dynamics that control such a deviant behaviour. In chapter 10, Pascal Perez and colleagues describe a first attempt to simulate illicit drug use and local markets in Australia. Authors admit that blending together law enforcement, harm reduction, and treatment strategies already represents a daunting challenge; but, trying to infer users' or dealers' behavioural patterns - elusive and secretive by nature - needs to be dealt with through trans-disciplinary and consensual approaches.

On the other hand, unpredictable non-linear interactions are the raison d'etre of complex adaptive systems. The self-referential problem proposed by Arthur (1994) can drive a system to an equivalent situation to the one described by Durkheim. But in the El Farol case, individual behaviours are perfectly deterministic while totally unpredictable for an external observer. Conversely, some perfectly predictable individual behaviours interacting together can lead to unpredictable global behaviour of the whole system. In chapter 11, David Batten and George Grozev provide a clear illustration of the impact of such non-linear interactions in the case of the Australian National Electricity Market (NEM). They describe the NEM as an evolving system of complex interactions between 
human behaviour in markets, technical infrastructures and the natural environment and propose to explore plausible sustainable futures through agent-based simulations.

In chapter 3, Pascal Perez insists on the inherent uncertainty attached to human ecosystems. He argues that scientists studying these complex adaptive systems fall into two broad categories based on their epistemological postures: Legalists believe in a positivist and experimental science, while Revolutionaries claim that human ecosystems can only be apprehended through a post-normal approach to science. Drawing upon recent theoretical and methodological advances in agent-based modelling, he argues that an uncertainty-compliant science needs to overcome this dichotomy.

\section{Scales and hierarchies}

In their 2002 book entitled Panarchy: Understanding Transformations in Human and Natural Systems, Gundarson and Holling proposed a holistic and history contingent view of human ecosystems. The panarchy concept describes how a healthy system can invent, experiment, and survive through hierarchies and adaptive cycles that represent ecosystems and socio-ecosystems across scales. Each level of the panarchy operates at its own pace, protected from above by slower, larger levels but invigorated from below by faster, smaller cycles of innovation. The whole panarchy is therefore both creative and conserving. The interactions between adaptive cycles in a panarchy combine learning with continuity (Holling 2001). An obvious strength of the approach is to allow us to view resource management in a more structured way.

Indisputably, this theoretical framework helps clarify the meaning of sustainable development. But critics of the approach point out the direct filiation of panarchy from ecological modelling, in particular dynamical system modelling (DSM). While biological cycles are easy to define, much remains to be said about cycles within human societies. Stepp et al. (2003) provide a compelling list of human characteristics hardly tractable at the level of societies or human ecosystems. The way forward lies in our ability to transcend boundaries between systemic and atomistic approaches. In chapter 13, David McDonald and colleagues demonstrate that it is technically possible to blend system dynamics and individual (or group) behaviours of complex marine environments. In an entirely different context, Ang Yang and colleagues (chapter 8) analyse interconnections between several communication, observation and command networks on a battlefield in order to infer relevant conclusions for modern warfare, including fallible human judgement. 


\section{Exploring human ecosystems with agents}

\section{Autonomous and adaptive agents}

For some years, the fields of software engineering and artificial intelligence have been making use of the concept of interacting autonomous agents. Although the term agent has been defined and used in several ways by other scientific groups, for the purposes of software engineering it has been sufficient to start with a minimal definition of an agent, such as the following (Ferber 1999): an agent is a physical or virtual entity that:

- is capable of acting in an environment;

- can communicate directly with other agents;

- is driven by an autonomous set of individual goals or objectives;

- possesses resources of its own;

- is capable of perceiving its environment to some extent;

- has only a partial representation of this environment;

- possesses skills and can offer services;

- may be able to reproduce itself; or

- whose behaviour tends towards satisfying its objectives, taking account of the resources and skills available to it.

The definition corresponds closely to a kind of living organism whose behaviour is aimed at satisfying its own needs and fulfilling its own objectives. Adaptation among such an assembly of agents may occur in two ways: by altering individual characteristics (learning), or as a collective process that brings reproductive processes into play (evolution). We can think of this combination as adaptation that is simultaneously individual and collective. A broad range of agents that are simulated in several different contexts, as described in the second part of this book, are summarised in the Table 1.1.

\section{Table 1.1. Agents simulated in Chapters 7-14}

\begin{tabular}{llll}
\hline Chapter & Author(s) & Simulation & Types of agents \\
\hline 7 & Daniell et al. & Housing market & House occupants or households \\
8 & Yang et al. & Warfare model & Command and control groups \\
9 & Elliston and Beare & Pest incursion & Pests, farmers, contractors \\
10 & Perez et al. & Illicit drug market & $\begin{array}{l}\text { Users, dealers, wholesalers, police and outreach } \\
\text { workers }\end{array}$ \\
11 & Batten and Grozev & Electricity market & $\begin{array}{l}\text { Generator firms, retailers, network service providers, } \\
\text { customers and traders }\end{array}$ \\
12 & Dray et al. & Atoll water & Landowners management \\
13 & McDonald et al. & Coastal marine & $\begin{array}{l}\text { Fishing, shipping, ecosystems; petroleum, environ- } \\
\text { ment }\end{array}$ \\
& & Rangeland & Pastoral enterprises
\end{tabular}




\section{Hierarchies of autonomous agents}

In his quest to find a general biology, Stuart Kauffman suggests that the biosphere got itself constructed 'by the emergence and persistent co-evolution of autonomous agents' (Kauffman 2000: 3). He goes further by suggesting that there may be a fourth law of thermodynamics that roughly states that biospheres maximise the average secular construction of the diversity of autonomous agents and the ways those agents make a living to propagate further. Although this raises a labyrinth of issues about the core features of autonomous agents and their abilities to manipulate the world on their own behalf, it also has important ramifications for the study of human ecosystems. Complex webs of interacting life within ecosystems need to be recognised and addressed in any simulation that attempts to explore alternative co-evolutionary pathways for the future.

Kauffman defines an autonomous agent as a self-reproducing system able to perform at least one thermodynamic work cycle. If true, this means that all freeliving cells and organisms are autonomous agents. It also suggests that we lack a concept of propagating organisation. Complexity in organisation rises from the subatomic through the atomic, molecular, cellular, organismal, societal, and ecological system levels. Coevolving autonomous agents may be co-constructing and propagating organisations of work simultaneously at all of these levels. This poses a major challenge for our new kind of science. Nevertheless, more ambitious simulation models such as the Management Strategy Evaluation (MSE) approach for the North West Shelf region (see chapter 14) are beginning to grapple with several levels of the agent hierarchy.

\section{Interconnected and embedded agents}

The time when systemic Dynamical System Modelling (DSM) and atomistic (Agent-Based Modelling (ABM)) approaches were described as inherently conflicting is gone. Pioneering work has proven that these approaches are largely complementary (Carpenter et al. 1999; Janssen et al. 2000). DSM provides an elegant analytical framework to study dynamic equilibrium of a system, to the extent that the global parameters can be made explicit. On the other hand, ABM demonstrates an ability to demonstrate emergent phenomena, but is not a predictive tool. Hence, ABM could help discover some emerging values of systemwide parameters and DSM might provide bounding behavioural domains for agent-based simulations.

In chapter 5, David Newth describes how, from social systems to computer networks, graphs can be used to describe the way in which components in large systems interact. In mathematical terms, a network is represented as a graph, in which nodes represent network elements and edges define relationships between elements. According to the author, this powerful paradigm now needs to be 
coupled to agent-based approaches in order to refine our understanding of human ecosystems.

A comprehensive series of research tends to illustrate how social network structure can be used to determine the characteristics of individual actors, and how social network structure and associated dynamics can modify the behaviour of these actors (Borgatti and Foster 2003). But much remains to be said about the influence of autonomous and partly rational actors on the structure and evolution of these social networks. Here lies the crucial question of how social coalitions and factions form and dissolve over time. Coming back to our previous analogy between human body integrity and environmental sustainability, it is a matter of knowing how system structure and fuctions co-evolve in order to maintain the resilience of a given human ecosystem.

In chapter 9, Lisa Elliston and Steve Beare illustrate the impact of such an organic link with their simulation of a pest invasion in Northern Queensland. Ways in which wheat farmers and contractors interact shape the network over which Karnal bunt, a disease of wheat, diffuses and contaminates various parts of the countryside. Conversely, the presence of contaminated areas influences the behaviour of local farmers and the strategies deployed by the Quarantine services. In a different context, Pascal Perez and colleagues (chapter 10) describe an equivalent co-evolutionary process of interactions between illicit drug markets and law enforcement strategies. So called hot spots move around the urban environment due to endless adaptive strategies deployed by police forces and crime syndicates.

\section{Exploring national research priorities with agents}

The second part of this book provides meaningful examples of agent-based modelling applications. Ranging from warfare strategies to natural resources management, the different chapters display two characteristics that, too often, are lacking in descriptions of computer simulation. First, the context of application is carefully presented, as it seems essential to understand the reasons why decision-makers decided to fund or to participate in such projects. Then, the corollary is to give the opportunity to decision-makers or practitioners to comment on the usefulness of the approach. Hence, each chapter is introduced by a specific foreword from a personality who was actively involved or interested in using the outcomes of the modelling process.

Effective applied research doesn't need only to be deeply rooted in a context and to be accessible to stakeholders, it also needs to participate in the global backing of national capacities. In Australia, the National Research Priorities (NRPs) provide a general framework guiding research institutions. Four NRPs are currently developed: an environmentally sustainable Australia; promoting 
and maintaining good health; frontier technologies for transforming Australian industries; and safeguarding Australia.

One could argue that agent-based modelling and computer simulation are breakthrough sciences that deserve to fall entirely into the third priority. On the contrary, the variety of contexts discussed in this book demonstrate the large range of applications of these methods by linking each chapter of the second part of this book to a relevant NRP.

\section{An environmentally sustainable Australia}

\section{Water-a critical resource}

In chapter 12, Anne Dray and colleagues describe how they used multi-agent simulations in conjunction with a role playing game to develop a Negotiation Support System for groundwater management in Tarawa (Republic of Kiribati). Their Companion Modelling approach relies on three successive stages. First, a Global Targeted Appraisal focuses on social group leaders in order to collect different standpoints and their articulated mental models. Then, these contrasted models are merged into a single conceptual one using UML (Unified Modelling Language) formalism. This conceptual model is further simplified in order to create a role-playing game. This computer-assisted game is played during iterative sessions, generating innovative rules and water management scenarios among stakeholders.

\section{Transforming existing industries}

Ryan McAllister and colleagues (chapter 14) present an interesting reflection on Australian pastoral land-use systems that have been characterised by privateproperty regimes, which, to varying degrees, have created fragmented and disconnected landscapes. The authors argue that there are both environmental and economic risks associated with productive land fragments being too small, and these risks necessitate an understanding of fragmentation's driving forces. Understanding these forces, however, is made difficult because the problem involves social, economic and environmental factors, interacting over a range of temporal and spatial scales. Hence, the authors developed an agent-based model to explore rangeland dynamics that involve such complexity. The current model contains pastoralists, livestock, key ecological processes, and governance.

\section{Sustainable use of Australia's biodiversity}

In chapter 13, David McDonald and colleagues propose a Management Strategy Evaluation (MSE) framework to demonstrate practical science-based methods that support integrated regional planning and management of coastal and marine ecosystems of the North West Shelf region (Western Australia). Their multipleuse MSE has, so far, focused on four sectors: oil and gas, conservation, fisheries 
and coastal development. For each sector a selection of development scenarios, provided by the relevant interest groups, is represented. These scenarios include prospective future sectoral activities and their impacts, and the sectoral response to management policy and strategies. According to the authors, the agent-based modelling software InVitro is well placed for analysing prospective social and ecological impacts of multiple-use management strategies in a risk-assessment framework such as MSE.

\section{Promoting and maintaining good health}

\section{Strengthening Australia's social and economic fabric}

Katherine Daniell and colleagues (chapter 7) present a model that was used to evaluate the plausible futures of Christie Walk housing development in innercity Adelaide, Australia. The authors underline the fact that assessing the sustainability of development proposals has become of great importance to policy and decision makers. However, effective methods of assessing the overall sustainability of housing developments (proposed or existing) have yet to be established. This chapter presents a new methodology to assess the sustainability of housing development systems. The methodology uses a Sustainability Scale approach for livelihood indicators, coupled with multi-agent simulations to represent the complex housing patterns.

\section{Safeguarding Australia}

\section{Critical infrastructure}

In chapter 11, David Batten and George Grozev describe the development of an agent-based simulation model that represents Australia's National Electricity Market (NEM) as an evolving system of complex interactions between human behaviour in markets, technical infrastructures and the natural environment. This simulator, named NEMSIM, is the first of its kind in Australia. Users will be able to explore various evolutionary pathways of the NEM under different assumptions about trading and investment opportunities, institutional changes and technological futures, including alternative learning patterns as participants grow and change. Simulated outcomes can help the user to identify futures that are eco-efficient - for example, maximising profits in a carbon-constrained future.

\section{Protecting Australia from invasive diseases and pests}

Lisa Elliston and Steve Beare (chapter 9) present a model investigating the impact of a potential incursion of Karnal bunt in wheat in a valuable agricultural producing region of Australia. An incursion management model was developed to estimate the regional economy effects of the exotic pest incursion in the agricultural sector. By developing an agent-based spatial model that integrates the 
biophysical aspects of the disease incursion with the agricultural production system and the wider regional economy, the model can be used to analyse the effectiveness and economic implications of alternative management strategies for a range of different incursion scenarios.

\section{Protecting Australia from terrorism and crime}

In chapter 10, Pascal Perez and colleagues have explored the complexity of illicit drug markets in Melbourne. The intricacy of multiple interactions between individuals, the various time lines linked to different aspects of harm reduction, and contrasted social rationalities observed among field practitioners (prevention, law enforcement, harm reduction) contribute to the creation of complex and unpredictable systems. In order to explore this complexity, an agent-based model called SimDrug was designed. The prototype model includes users, dealers, wholesalers, outreach workers and police forces. In order to overcome the limited knowledge we have of underground illicit activities, a trans-disciplinary group of experts regularly informed (and validated) the model with consensual rules.

\section{Transformational defence technologies}

Ang Yang and colleagues (chapter 8) bring us into the complex world of warfare for which a number of Agent-Based Distillation Systems (ABDs) have been developed and adopted to study the dynamics of warfare and gain insight into military operations. According to the authors, these systems have facilitated the analysis and understanding of combat. However, these systems are unable to meet the new needs of defense, arising from current practice of warfare and the emergence of the theory of Network Centric Warfare (NCW). The authors propose a network centric model which provides a new approach to understand and analyse the dynamics of both platform centric and network centric warfare.

\section{Building a new science for a complex world}

\section{Towards holistic visions and polymorphic tools}

\section{Holistic visions}

To develop a truly holistic view of human ecosystems - to identify feasible pathways towards their sustainability - it is necessary to make use of tools and techniques that are able to grapple with the complex interactions between human activities and the stability or resilience of the non-human ecosystem habitat. Various chapters in this book show that human behaviour can be complex in itself, necessitating its representation in a more sophisticated manner in computational approaches that aim to improve our understanding of the collective outcomes under alternative management strategies. Agent-based modelling is one promising way of exploring the possible collective implications of more 
complicated behaviour of human agents in an ecosystem context. Role-playing games are another valuable method of engaging with stakeholders and eliciting their views in an ecosystem context.

Opportunities for further, impressive progress exist. Recent developments in object-oriented programming languages have allowed users to create autonomous modules which can interact with each other even when they have been designed by different people, different teams or different companies (Ferber 1999). Agentbased models and Multi-Agent Systems (MAS) have an important role to play in this endeavour by serving as possible successors to object-oriented systems and combining local behaviours with autonomy, best-practice agent modules and distributed decision making. Thus it seems very likely that the software engineering of tomorrow for addressing more complex societal problems will be agent-oriented, just as that of today is object-oriented.

\section{Polymorphic tools}

To make our new kind of science truly polymorphic, the mapping of relationships between agents needs to be continuously updated depending on circumstances, proficiencies, perceptions, tasks to accomplish, or relational rules based on the social contracts established. This means that the simulation models need to recognise different views, biases or expertise. Such simulation tools are more participatory in nature, and should lead to further exciting developments in the future.

In the field of companion modelling, for example, convergent views may emerge after allowing several recognised experts to take the lead one after the other. This is similar to a cooperative team sport like football; for instance, the rightwinger becomes the leader when the ball comes into his space, but it can happen that he becomes the goalkeeper when the situation requires it. This flexible kind of companion modelling forms the basis of AtollGame (chapter 12), in which the agent-based model and the corresponding role-playing game were designed according to different viewpoints - converging or conflicting - that were recorded during a series of interviews.

\section{Towards a new epistemology of science}

\section{Integration and implementation}

The sixth chapter of this book presents an argument by Gabriele Bammer to think differently about the future of current science and the way it engages with decision-makers. According to the author, developing a new specialisation-Integration and Implementation Sciences - may be an effective way to draw together, and significantly strengthen, the theory and methods necessary to tackle complex societal issues and problems. It would place research on human ecosys- 
tems in broader context and link it with a range of complementary concepts and skills.

There is indeed a pressing need to integrate not only the participation, but, more importantly, the engagement of local stakeholders in projects that concern their future. Aslin and Brown (2004) argue that local communities need to be involved in the analysis of the results (consultation) and the choice of the possible scenarios (participation), but also in the knowledge creation itself (engagement). This is a post-normal posture adopted, for example, within companion modelling approaches (Bousquet et al. 2002), for which Anne Dray and colleagues (chapter 12) provide a good illustration.

Research on sustainable development too often relies on deductive scientific approaches to reach outcomes that require more inductive and flexible solutions. But flexibility means that one must assume some uncertainty during implementation and must get away from traditional reliance on deterministic and predictable solutions. What is true at the technical level becomes paramount at the political level. As stated by Bradshaw and Borchers (2000: 1):

One of the most difficult aspects of translating science into policy is scientific uncertainty. Whereas scientists are familiar with uncertainty and complexity, the public and policy makers often seek certainty and deterministic solutions.

This call for a meta-integration of science takes us back to the beginning of this book. Roger Bradbury's vision of a complex and adaptive science is nothing less than a fantastic opportunity for scientists to think differently about the goals of their own research and their relations to others.

\section{A few last words}

We hope that you will enjoy reading such a diverse ouvrage whose purpose is to attract more early career scientists into our field of research and to convince decision-makers that a growing contingent of colleagues working on complexity theory can provide useful tools and methods to better understand complex and adaptive environments. It is time to reassure you, the reader, that the rise of a Complex Science for a Complex World doesn't mean more complicated relationships between science and society.

\section{References}

Arthur, W.B. (1994) Inductive behaviour and bounded rationality. The American Economic Review 84: 406-11.

Aslin, H.J. and V.A. Brown (2004) Towards whole of community engagement:

A practical toolkit. Canberra, Australia: Murray-Darling Basin Commission. 
Batten, D.F. (2000) Discovering Artificial Economics. How Agents Learn and Economies Evolve. Oxford: Westview Press.

Borgatti S.P. and P.C. Foster (2003) The network paradigm in organizational research: a review and typology. Journal of Management 29(6): 991-1013.

Bousquet F., O. Barreteau, P. d'Aquino, M. Etienne, S. Boisseau, S. Aubert, C. Le Page, D. Babin and J.C. Castella (2002) Multi-agent systems and role games: collective learning processes for ecosystem management. In M. A. Janssen (ed.) Complexity and Ecosystem Management: The Theory and Practice of Multi-agent Systems, pp. 248-85. Cheltenham: Edward Elgar.

Bradshaw, G.A. and J.G. Borchers (2000) Uncertainty as information: narrowing the science-policy gap. Conservation Ecology 4(1): 7. Available at http://www.consecol.org/vol4/issl/art7/

Carpenter, S., W. Brock and P. Hanson (1999) Ecological and social dynamics in simple models of ecosystem management. Conservation Ecology 3(2): 4. Available at http://www.consecol.org/vol3/iss2/art4

Dawkins, R. (1976) The Selfish Gene. Oxford: Oxford University Press.

Downs, A. (1962) The law of peak-hour expressway congestion. Traffic Quarterly 16: 393-409.

Durkheim, E. (1979) Suicide: A Study in Sociology. Transl. J.A. Spaulding, G. Simpson. New York: Free Press.

Ferber, J. (1999) Multi-Agent Systems: An Introduction to Distributed Artificial Intelligence. New York: Addison Wesley.

Gunderson, L.H. and C.S. Holling (eds) (2002) Panarchy: Understanding Transformations in Human and Natural Systems. Washington, D.C.: Island Press.

Holling, C.S. (2001) Understanding the Complexity of Economic, Ecological, and Social Systems. Ecosystems 4: 390-405.

Holland, J.H. (1995) Hidden Order: How Adaptation Builds Complexity. New York: Helix Books (Addison Wesley).

Janssen M.A., B.H. Walker, J. Langridge and N. Abel (2000) An adaptive agent model for analysing co-evolution of management and policies in a complex rangeland system. Ecological Modelling 131: 249-68.

Kauffman, S.A. (2000) Investigations. New York: Oxford University Press.

Lansing, J.S. (2003) Complex adaptive systems. Annual Review of Anthropology 32: $183-204$.

Schrodinger, E. (1967) What is life? With Mind and Matter and Autobiographic Sketches. Cambridge, UK: Cambridge University Press. 
Stepp, J.R., E.C. Jones, M. Pavao-Zuckerman, D. Casagrande, and R.K. Zarger (2003) Remarkable properties of human ecosystems. Conservation Ecology 7(3): 11. Available at http://www.consecol.org/vol7/iss3/art1 1 